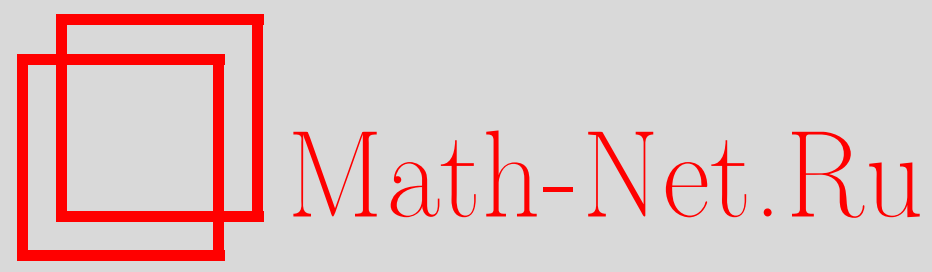

Р. В. Кожан, Асимптотика собственных значений двухдиагональных матриц Якоби, Матем. заметки, 2005, том 77, выпуск 2, 313-316

DOI: https://doi.org/10.4213/mzm2492

Использование Общероссийского математического портала Math-Net.Ru подразумевает, что вы прочитали и согласны с пользовательским соглашением http://www . mathnet.ru/rus/agreement

Параметры загрузки:

IP : 54.209 .52 .79

26 апреля 2023 г., $14: 28: 34$

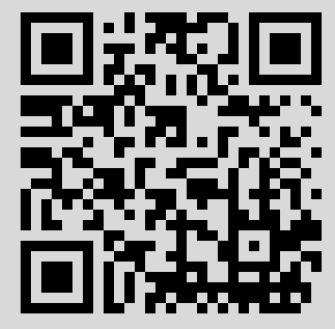




\section{АСИМПТОТИКА СОБСТВЕННЫХ ЗНАЧЕНИЙ ДВУХДИАГОНАЛЬНЫХ МАТРИЦ ЯКОБИ}

\section{P. В. Кожан}

1. Введение. Рассмотрим в гильбертовом пространстве $H:=\ell_{2}(\mathbb{N})$ со скалярным произведением $(\cdot \mid \cdot)$ оператор Якоби

$$
J=S A+A S^{*},
$$

где $S$ - оператор сдвига вправо, а $A$ - диагональный оператор с диагоналюю $\left(a_{n}\right)_{n=1}^{\infty}$, т.е.

$$
S e_{n}=e_{n+1}, A e_{n}=a_{n} e_{n}, \quad n \in \mathbb{N} .
$$

Здесь $\left(e_{n}\right)_{n=1}^{\infty}-$ стандартный базис в $\ell_{2}(\mathbb{N})$. Относительно числовой последовательности $\left(a_{n}\right)_{n=1}^{\infty}$ мы предполагаем, что она состоит из положительных чисел, для которых выполняется условие

$$
\lim _{n \rightarrow \infty} \frac{a_{n+1}}{a_{n}}=0 .
$$

Оператор $J$ является компактным и самосопряженным, а все его собственные значения простые и отличные от нуля. При этом точечный спектр является симметричным относительно нуля, т.е. $\sigma_{p}(J)=\left\{ \pm \lambda_{n} \mid n \in \mathbb{N}\right\}$, где последовательность $\left(\lambda_{n}\right)_{n=1}^{\infty}$ состоит из положительных чисел, которые монотонно стремятся к нулю.

Цель нашей работы заключается в изучении асимптотического поведения последовательности $\left(\lambda_{n}\right)_{n=1}^{\infty}$. Постановка такой задачи возникла в связи с работой [1], где изучалось асимптотическое поведение последовательности $\left(\lambda_{n}\right)_{n=1}^{\infty}$ в случае, когда $a_{n}=q^{n^{s}}$ (где $\left.q \in(0,1), s>0\right)$, и было доказано, что $\lambda_{n}$ удовлетворяют условию $a_{2 n-1}>\lambda_{n}>a_{2 n+1}$. Основным результатом нашей работы является следующая

Теорема 1.1. Если выполнено условие (1.2), то

$$
\lambda_{n}=(1+o(1)) a_{2 n-1}, \quad n \rightarrow \infty .
$$

Отметим, что наш подход базируется на изучении квадратичной формы оператора $J$ и является более элементарным, чем подход в [1], который использовал аппарат теории целых функций.

2. Доказательство теоремы 1.1. Пусть $P_{n}: H \rightarrow H$-ортопроектор на подпространство

$$
H_{n}=\left\{x \in H \mid x=\left(x_{k}\right)_{k=1}^{\infty} \forall k>n: x_{k}=0\right\}, \quad n \in \mathbb{N}
$$

Положим

$$
J_{n}:=P_{n} J P_{n}
$$

Обозначим также через $T_{n}$ сужение оператора $J_{n}$ на пространство $H_{n}$.

Лемма 2.1. Спектры операторов $J$ и $J_{n}, n \in \mathbb{N}$, симметричны относительно нуля. При этом все собственные значения оператора $J$ и все ненулевые собственные значения операторов $J_{n}$ являются простыми. Кроме того, число $\lambda=0$ не является собственным значением оператора $J$.

ДоКАЗАТЕЛЬСТво. Обозначим через $U$ диагональный оператор, который действует по формуле

$$
U e_{n}=(-1)^{n} e_{n}, \quad n \in \mathbb{N} .
$$

Поскольку $U^{-1} S U=-S$ и $U^{-1} A U=A$, то

$$
U^{-1} J U=-J, \quad U^{-1} J_{n} U=-J_{n},
$$

откуда следует симметричность спектров операторов $J$ и $J_{n}$. Доказательство остальных утверждений леммы очевидно.

Обозначим через $\left(\lambda_{k}\right)_{k=1}^{\infty}\left(\right.$ соответственно $\left.\left(\lambda_{k}^{(n)}\right)_{k=1}^{\infty}\right)$ последовательность неотрицательных собственных значений оператора $J$ (соответственно $J_{n}$ ), занумерованную в убывающем порядке с учетом кратности. Через $\left(s_{k}(X)\right)_{k=1}^{\infty}$ мы будем обозначать последовательность $s$-чисел компактного оператора $X: H \rightarrow H$ (см. [2]). 
Лемма 2.2. Для произвольных $n \in \mathbb{N}$ выполняется равенство

$$
\prod_{k=1}^{n} \lambda_{k}^{(2 n)}=\prod_{k=1}^{n} a_{2 k-1}
$$

ДокАЗАТЕльство. Учитьвая лемму 2.1 , получаем, что

$$
\prod_{k=1}^{n} \lambda_{k}^{(2 n)}=\left|\operatorname{det} T_{2 n}\right|^{1 / 2} .
$$

Простые подсчеты дают, что для произвольных $n>1$

и тем самым лемма доказана.

$$
\operatorname{det} T_{2 n}=-a_{2 n-1}^{2} \operatorname{det} T_{2 n-2}
$$

Лемма 2.3. Для достаточно больших $n_{0} \in \mathbb{N}$ выполняются неравенства

$$
\begin{aligned}
& \left\|J_{m}-J_{n}\right\| \leqslant a_{n}+a_{n+1}, \quad m>n \geqslant n_{0}, \\
& \left\|J-J_{n}\right\| \leqslant a_{n}+a_{n+1}, \quad n \geqslant n_{0} .
\end{aligned}
$$

ДоКАЗАТЕЛЬСтво. Из условия (1.2) следует, что $a_{n+1} \leqslant a_{n}$, начиная с некоторого $n_{0} \in \mathbb{N}$, и, следовательно, для $n>n_{0}$ и $\|x\|=1$ получим

$$
\begin{aligned}
& \left|\left(\left(J-J_{n}\right) x \mid x\right)\right| \leqslant 2\left|\sum_{k=n}^{\infty} a_{k} x_{k} \overline{x_{k+1}}\right| \leqslant \sum_{k=n}^{\infty} a_{k}\left(\left|x_{k}\right|^{2}+\left|x_{k+1}\right|^{2}\right) \\
& \quad=\sum_{i=0}^{\infty} a_{n+2 i}\left(\left|x_{n+2 i}\right|^{2}+\left|x_{n+2 i+1}\right|^{2}\right)+\sum_{i=0}^{\infty} a_{n+2 i+1}\left(\left|x_{n+2 i+1}\right|^{2}+\left|x_{n+2 i+2}\right|^{2}\right) \\
& \quad \leqslant a_{n}+a_{n+1} .
\end{aligned}
$$

Таким образом, (2.2) доказано. Аналогично доказьвается оценка (2.1).

ЛЕмма 2.4. Неравенство

$$
\lambda_{k}^{(2 k)} \geqslant \frac{a_{2 k-1}}{2}
$$

выполняется для бесконечного количества номеров $k \in \mathbb{N}$.

ДокАЗАТЕЛЬСтво. Проведем доказательство от противного: пусть существует $n_{1} \in \mathbb{N}$ такое, что $\lambda_{k}^{(2 k)}<a_{2 k-1} / 2$ для всех $k \geqslant n_{1}$. Согласно условию (1.2) существует $n_{2} \in \mathbb{N}$ такое, что $a_{n+1} \leqslant a_{n} / 8$ для всех $n \geqslant n_{2}$. Возьмем $p=\max \left\{n_{1}, n_{2}\right\}$, и пусть $N>p$. Принимая во внимание лемму 2.2 , имеем

Так как

$$
\prod_{i=1}^{N} a_{2 i-1}=\prod_{i=1}^{N} \lambda_{i}^{(2 N)}=\prod_{i=1}^{p} \lambda_{i}^{(2 N)} \cdot \prod_{i=p+1}^{N} \lambda_{i}^{(2 N)}
$$

учитывая лемму 2.3 , имеем

$$
\left|\lambda_{i}^{(2 N)}-\lambda_{i}^{(2 i)}\right|=\left|s_{2 i}\left(J_{2 n}\right)-s_{2 i}\left(J_{2 i}\right)\right| \leqslant\left\|J_{2 N}-J_{2 i}\right\|,
$$

$$
\lambda_{i}^{(2 N)}<\lambda_{i}^{(2 i)}+a_{2 i}+a_{2 i+1} \leqslant \frac{a_{2 i-1}}{2}+2 \cdot \frac{a_{2 i-1}}{8}=\frac{3 a_{2 i-1}}{4}, \quad p<i \leqslant N .
$$

Поскольку

из (2.4) получаем

$$
\left|\lambda_{i}^{(2 N)}\right| \leqslant\left\|J_{2 N}\right\|=\left\|P_{2 N} J P_{2 N}\right\| \leqslant\|J\|, \quad 1 \leqslant i \leqslant p,
$$

$$
\prod_{i=1}^{N} a_{2 i-1} \leqslant\|J\|^{p} \prod_{i=p+1}^{N} \frac{3 a_{2 i-1}}{4}
$$

и, следовательно,

$$
\prod_{i=1}^{p} a_{2 i-1} \leqslant\|J\|^{p}\left(\frac{3}{4}\right)^{N-p} .
$$

Устремляя теперь $N$ к $+\infty$, получаем, что среди чисел $a_{n}$ есть нулевые. Полученное противоречие заканчивает доказательство. 
ДоКАЗАТЕЛЬСТво ТЕОРЕмЫ 1.1. Зафиксируем произвольное $\varepsilon \in(0,1 / 8)$. Тогда существует (см. (1.2)) $n_{1} \in \mathbb{N}$ такое, что $a_{n} \leqslant \varepsilon a_{n-1}$ для всех $n \geqslant n_{1}$.

Сначала покажем, что при больших $n \in \mathbb{N}$ выполняется неравенство

$$
\lambda_{n} \leqslant(1+\varepsilon) a_{2 n-1} \text {. }
$$

Действительно, согласно с (2.2) существует $n_{0}>n_{1}$, для которого

$$
\left\|J-J_{2 n-1}\right\| \leqslant a_{2 n-1}+a_{2 n}, \quad n>n_{0} .
$$

Легко видеть, что $\lambda_{n}=s_{2 n}(J)$ и $s_{2 n}\left(J_{2 n-1}\right)=0$. Поэтому при $n>n_{0}$ имеем

$$
\lambda_{n}=s_{2 n}(J)-s_{2 n}\left(J_{2 n-1}\right) \leqslant\left\|J-J_{2 n-1}\right\| \leqslant a_{2 n-1}+a_{2 n} \leqslant(1+\varepsilon) a_{2 n-1} .
$$

Теперь убедимся, что для достаточно больших $n \in \mathbb{N}$ выполняется неравенство

$$
\lambda_{n} \geqslant(1-2 \varepsilon) a_{2 n-1} .
$$

Исходя из результата леммы 2.4 , фиксируем $m>n_{1}$ такое, что $\lambda_{m}^{(2 m)} \geqslant a_{2 m-1} / 2$. Поскольку $\lambda_{i}^{(2 m)} \geqslant \lambda_{m}^{(2 m)} \geqslant a_{2 m-1} / 2, i=1, \ldots, m$, то, как следует из спектральной теоремы,

$$
\left(J_{2 m} x \mid J_{2 m} x\right) \geqslant \frac{1}{4} a_{2 m-1}^{2} \sum_{k=1}^{2 m}\left|x_{k}\right|^{2}, \quad x \in H_{2 m} .
$$

Для произвольного $r>m+1$ рассмотрим пространство

$$
G_{2 r}^{m}:=\left\{\left(x_{n}\right)_{n=1}^{\infty} \in H_{2 r} \mid x_{2 m+1}=0\right\} .
$$

Заметим, что $\operatorname{dim} G_{2 r}^{m}=2 r-1$. С помощью непосредственных вычислений получаем, что для произвольных $x \in G_{2 r}^{m}$

$$
\begin{aligned}
(J x \mid J x)= & \left(J_{2 m} x \mid J_{2 m} x\right)+a_{2 m}^{2}\left|x_{2 m}\right|^{2} \\
& +\sum_{k=2 m+2}^{2 r}\left(a_{k}^{2}+a_{k-1}^{2}\right)\left|x_{k}\right|^{2}+2 \operatorname{Re}\left(\sum_{k=2 m}^{2 r-2} a_{k} a_{k+1} x_{k} \overline{x_{k+2}}\right) .
\end{aligned}
$$

Поскольку $a_{k}<\varepsilon a_{k-1}$ для всех $k \geqslant 2 m$, то

$$
2\left|a_{k} a_{k+1} x_{k} \overline{x_{k+2}}\right| \leqslant 2 \varepsilon\left|a_{k-1} x_{k}\right| \cdot\left|a_{k+1} x_{k+2}\right| \leqslant \varepsilon\left(a_{k-1}^{2}\left|x_{k}\right|^{2}+a_{k+1}^{2}\left|x_{k+2}\right|^{2}\right), \quad k \geqslant 2 m,
$$

и, следовательно,

$$
2 \operatorname{Re}\left(\sum_{k=2 m}^{2 r-2} a_{k} a_{k+1} x_{k} \overline{x_{k+2}}\right) \geqslant-\varepsilon a_{2 m-1}^{2}\left|x_{2 m}\right|^{2}-2 \varepsilon \sum_{k=2 m+2}^{2 r} a_{k-1}^{2}\left|x_{k}\right|^{2} .
$$

Отсюда на основании (2.8) и (2.7) получаем, что

$$
(J x \mid J x) \geqslant \frac{a_{2 m-1}^{2}}{4} \sum_{k=1}^{2 m}\left|x_{k}\right|^{2}-\varepsilon a_{2 m-1}^{2}\left|x_{2 m}\right|^{2}+(1-2 \varepsilon) \sum_{k=2 m+2}^{2 r} a_{k-1}^{2}\left|x_{k}\right|^{2} .
$$

Поскольку $\varepsilon \in(0,1 / 8)$ и $r>m+1$, то $a_{2 m-1}^{2}(1 / 4-\varepsilon) \geqslant a_{2 r-1}^{2}$, откуда, учитьвая $(2.9)$, получаем

$$
(J x \mid J x) \geqslant(1-2 \varepsilon) a_{2 r-1}^{2} \sum_{k=1}^{2 r}\left|x_{k}\right|^{2}, \quad x \in G_{2 r}^{m}
$$

Применяя теперь принцип минимакса [3, гл. 8] с учетом леммы 2.1 и того, что $\operatorname{dim} G_{2 r}^{m}=2 r-1$, получаем

$$
\lambda_{r} \geqslant(1-2 \varepsilon)^{1 / 2} a_{2 r-1} \geqslant(1-2 \varepsilon) a_{2 r-1}, \quad r>m+1 .
$$

Тем самым, неравенство (2.6) доказано. Из (2.5) и (2.6) очевидным образом следует справедливость утверждения теоремы 1.1 . 


\section{СПИСОК ЦИТИРОВАННОЙ ЛИТЕРАТУРЫ}

1. Тур Э. А. // Матем. заметки. 2003. Т. 74. № 3. С. 449-462. 2. Гохберг И. Ц., Крейн М. Г. Введение в теорию линейных несамосопряженных операторов. М.: Наука, 1965. 3. Рид М., Саймон Б. Методы современной математической физики. Т. 4. М.: Мир, 1982. 